\title{
Procedimentos dolorosos agudos no recém-nascido pré-termo em uma unidade neonatal
}

\author{
Acute painful procedures pain in the preterm newborn in a neonatal unit \\ Procedimientos dolorosos agudos en el recién nacido pre-término en una unidad neonatal
}

\author{
Érica Célia Sousa Rocha'; Liana Albuquerque da Silva"; Marcelle Campos de Araujo"I; \\ Silvia Schoenau de Azevedo'v ; Maria de Fatima Junqueira-Marinhov
}

\begin{abstract}
RESUMO
Objetivo: analisar o número de procedimentos dolorosos agudos e manejo da dor em recém-nascidos pré-termo em uma unidade neonatal. Método: estudo descritivo, transversal. Teve como campo uma unidade neonatal de uma instituição pública de saúde. Os participantes da pesquisa foram recém-nascidos abaixo de 35 semanas. Resultados: foram incluídos 17 recémnascidos. Foi quantificado um total de 729 procedimentos dolorosos. Em média foram realizados 42,9 procedimentos por recém-nascido durante os primeiros 14 dias de vida, sendo aproximadamente três procedimentos realizados por bebê no serviço diurno. Dentre os procedimentos, o mais frequente foi a punção de calcâneo (23,9\%). O manejo predominante foi a contenção facilitada $(32,7 \%)$. A (re)inserção de pronga foi o segundo procedimento mais realizado. Conclusões: $O$ estudo permitiu apreender que o manejo da dor no recém-nascido pré-termo internado em uma unidade neonatal ainda é um desafio. Descritores: Dor; procedimentos clínicos; manejo da dor; recém-nascido.
\end{abstract}

\section{ABSTRACT}

Objective: to analyze the number of acute painful procedures and pain management in preterm infants in a neonatal unit. Method: a cross-sectional and descriptive study. It was conducted in a neonatal unit of a public health institution. The newborns younger than 35 weeks were the participants in the survey. Results: seventeen preterm newborns were included. A total of 729 painful procedures were quantified. On average, 42.9 procedures per newborn were performed during the first 14 days of birth, and approximately three procedures performed per day service by child. The most common procedure was the hell-stick (23.9\%). The predominant management was facilitated tucking (32.7\%). The CPAP prongs insertion/reinsertion was the second most performed procedure. Conclusions: The present study showed that the management of pain in the preterm newborns in a neonatal unit is strongly neglected by the professionals.

Descriptors: Pain; Clinical Procedures; Pain management; Newborn.

\section{RESUMEN}

Objetivo: analizar el número de procedimientos dolorosos agudos y manejo del dolor en recién nacidos prematuros en una unidad neonatal. Método: estudio descriptivo, transversal. Tuvo como campo una unidad neonatal de una institución pública de salud. Los participantes de la investigación fueron recién nacidos por debajo de 35 semanas. Resultados: se incluyeron 17 recién nacidos. Se cuantificó un total de 729 procedimientos dolorosos. En promedio se realizaron 42,9 procedimientos por recién nacido durante los primeros 14 días de vida, siendo acerca de tres procedimientos realizados por bebé en el servicio diurno. Entre los procedimientos, el más frecuente fue la punción de calcáneo (23,9\%). El manejo predominante fue la contención facilitada $(32,7 \%)$. La (re) inserción de prong fue el segundo procedimiento más realizado. Conclusiones: El estudio permitió aprehender que el manejo del dolor en el recién nacidos prematuros internado en una unidad neonatal se muestra fuertemente descuidado por el equipo.

Descriptores: Dolor; procedimientos clínicos; manejo del dolor; recién nacido.

\section{INTRODUÇÃO}

Os recém-nascidos (RN) admitidos na unidade de terapia intensiva neonatal (UTIN) são vulneráveis a procedimentos invasivos e potencialmente dolorosos aplicados de forma rotineira nessas unidades. Esses neonatos também estão expostos ao risco de seu estresse associado como resultado de procedimentos repetidos ${ }^{1}$.

Um estudo observacional realizado em um hospital da Holanda envolvendo 175 RN identificou que o número médio de procedimentos dolorosos por RN por dia foi igual a $11,4^{2}$.

\footnotetext{
'Enfermeira. Especialista em Enfermagem Neonatal. Aluna do Mestrado, Instituto Nacional de Saúde da Mulher, da Criança e do Adolescente Fernandes Figueira, Brasil. Email: erica.rocha14@hotmail.com

"Fisioterapeuta. Mestre. Instituto Nacional de Saúde da Mulher, da Criança e do Adolescente Fernandes Figueira, Brasil; E-mail: lianaasf@yahoo.com.br

II'Enfermeira. Mestre. Aluna do Doutorado, Instituto Nacional de Saúde da Mulher, da Criança e do Adolescente Fernandes Figueira, Brasil E-mail: enf.marcelle@gmail.com

IVEnfermeira. Especialista em Enfermagem Neonatal. Aluna do Mestrado, Universidade Federal de São Paulo. Brasil. E-mail: silvia_schoenau@yahoo.com.br

vpsicóloga. Doutora. Pesquisadora do Instituto Nacional de Saúde da Mulher, da Criança e do Adolescente Fernandes Figueira. Brasil. E-mail: mf.junqueira@terra.com.br
} 
Vale ressaltar que até pouco tempo, pensava-se erroneamente que a nocicepção era diminuída em recém-nascido pré-termo (RNPT) devido à imaturidade do sistema nervoso central (SNC). De fato, agora está claro que os receptores da pele e os nervos sensoriais periorais aparecem tão cedo quanto a 7ạ semana de gestação. Além disso, a imaturidade do SNC afeta preferencialmente as vias inibitórias descendentes que modulam as sinapses no corno dorsal da medula espinhal, que não aparecem até a $32^{\text {a }}$ semana de gestação ${ }^{3}$.

Cabe salientar que os procedimentos dolorosos desencadeiam uma série de respostas em todo o corpo, que incluem expressões faciais, mudanças na atividade cortical e ruptura da estabilidade fisiológica ${ }^{4}$.

Segundo a Academia Americana de Pediatria (2016), a prevenção e o manejo da dor em bebês devem ser o objetivo de todos os profissionais de saúde, e os membros da família devem receber orientação sobre esse assunto ${ }^{5}$.

Nas últimas três décadas, métodos para reduzir o estresse em bebês na UTIN tem sido alvo dos pesquisadores. Em sua prática assistencial, enfermeiros neonatais usam terapias como sucção não nutritiva, contato pele a pele (posição canguru realizada pela mãe), massagem, enrolamento e posicionamento para minimizar o estresse e promover o desenvolvimento ideal ${ }^{6}$.

Existe um corpo de literatura sobre a eficácia dessas intervenções e sugere que estas tenham efeitos positivos no estresse e uma variedade de resultados para bebês e pais ${ }^{7,8}$.

Porém, estudos revelam que a despeito do RN sentir dor, a capacidade e abordagem dos clínicos para avaliar e tratar a dor do RN é inadequada e controversa ${ }^{9}$.

Diante desse cenário de exposição frequente a procedimentos dolorosos que submetem o RNPT a consequentes repercussões a curto e longo prazo, o estudo objetivou analisar o número de procedimentos dolorosos agudos e o manejo da dor em RNPT em uma unidade neonatal.

\section{MÉTOdo}

Estudo observacional, transversal que teve como campo a área de atenção clínica de uma instituição pública localizada no Rio de Janeiro, composta por Unidade de Terapia Intensiva Neonatal (UTIN), Unidade de Cuidados Intermediários Convencional (UCINCO) e Unidade de Cuidados Intermediários Canguru (UCINCA). Os dados foram coletados no período de 27/06/2016 e 30/11/2016, apenas no serviço diurno (7:00h às 19:00h).

Foi utilizado um instrumento próprio para a contabilização dos procedimentos dolorosos, os quais foram escolhidos por meio de conversa com a enfermeira responsável pela rotina do setor e frente aos procedimentos mais corriqueiros nos primeiros 14 dias do RNPT em uma UTIN ${ }^{9}$. Nesta ficha constava a identificação do participante, o dia avaliado (do D1 ao D14), os procedimentos realizados e sua frequência no serviço diurno, e ao lado de cada procedimento continha espaço para anotação do respectivo manejo mencionado pela equipe.

Os procedimentos incluídos na ficha foram os classificados como muito dolorosos (punção venosa, punção arterial, punção de calcâneo, aspiração de tubo orotraqueal, intubação orotraqueal e cateter central de inserção periférica) e dolorosos (aspiração de vias aéreas superiores, re/inserção de pronga nasal, inserção de sonda gástrica e retirada de adesivos) $)^{9}$.

O procedimento de retirada de adesivo inicialmente não constava na ficha, sua inclusão ocorreu a partir de sugestão da própria equipe quando o projeto foi apresentado. Foi quantificada a retirada de qualquer tipo de adesivo, seja por fixações de sonda gástrica, tubo orotraqueal, punções venosas, curativos, e quaisquer outros que entrasse em contato direto com a pele do RNPT, e independente do tipo de material do adesivo (esparadrapo, fita microporosa, hidrococóide, filme transparente, entre outros).

O instrumento foi preenchido ao final do plantão através de perguntas realizadas pelas pesquisadoras, dirigidas à equipe (enfermeiros, técnicos de enfermagem, médicos e fisioterapeutas) sobre os procedimentos e seus respectivos manejos realizados durante o serviço diurno. Não houve caso de ausência de resposta por não recordação

Os participantes da pesquisa foram os recém-nascidos pré-termo (RNPT) das unidades de internação supracitadas, avaliados nos primeiros 14 dias de vida, que constituíram uma amostra de conveniência.

Critérios de exclusão: RNPT acima de 35 semanas de idade gestacional (IG) e/ou que apresentarem hemorragia intracraniana graus III e IV, síndromes genéticas, malformações do sistema nervoso, encefalopatia hipóxico-hisquêmica, alterações sensoriais (déficit auditivo e visual) e infecções congênitas do grupo TORCHS (sífilis, toxoplasma, citomegalovírus, rubéola e herpes) que evoluem com alteração do sistema nervoso central (SNC).

Esses critérios de exclusão se deram em função desta pesquisa fazer parte de outra mais ampla que visava avaliar o neurocomportamento dos RNPT, de modo que os critérios acima eram indicativos de exclusão. 
Também foram excluídos os RNPT que nasceram em outra instituição, os que foram transferidos para outro setor do hospital, outra instituição ou receberem alta durante o período de coleta de dados.

As variáveis de desfecho foram os procedimentos dolorosos agudos e o manejo da dor realizado pela equipe da unidade neonatal.

Já as variáveis de exposição foram: idade gestacional (IG), peso, sexo e o SNAPPE (Score for Neonatal Acute Physiology with Perinatal Extension version II) do RN.

O SNAPPE II avalia o paciente nas primeiras 12 horas de admissão na UTIN observando alterações fisiológicas múltiplas. A pontuação do SNAPPE II varia de zero a 162. Quanto maior a pontuação, maior a gravidade do $\mathrm{RN}^{11}$.

Com relação a possíveis fragilidades, acredita-se que o estudo pode apresentar um viés de memória, já que o instrumento utilizado para contabilização dos procedimentos dolorosos era preenchido ao final do plantão com perguntas dirigidas à equipe.

O estudo contou com uma amostra de conveniência que foi estabelecida pelo tempo de coleta de dados que aconteceu no período de cinco meses (entre junho e novembro de 2016).

O banco de dados foi criado através do programa Epi info versão 7.1.4.0 e a análise dos dados foi realizada através do programa SPSS versão 17.0, utilizando valores de média, mediana, percentil e desvio padrão. As variáveis categóricas foram analisadas utilizando o teste Qui-quadrado e as variáveis numéricas contínuas através da análise de variância (ANOVAs).

Todos os responsáveis (pais) pelos participantes foram esclarecidos quanto à pesquisa e quando em acordo assinaram o termo de consentimento livre e esclarecido para permitir a inclusão dos dados do RN no estudo. Não houve caso de recusa por nenhum dos pais para a pesquisa.

O projeto foi aprovado no Comitê de Ética em Pesquisa da instituição e possui CAEE número 50675915.1.0000.5269.

Foram admitidos, durante o período de coleta de dados, 117 RN, dos quais, 94 foram excluídos. Desses, 42 (44,7\%) foram excluídos por malformação do sistema nervoso central (SNC), 36 (38,3\%) por IG maior que 35 semanas, 10 (10,6\%) por síndromes genéticas e seis (6,4\%) por virem de outras unidades. Dos 23 RN incluídos, seis foram perdidos no decorrer da pesquisa, três por transferência, dois por instabilidade clínica e um dos RN desenvolveu Hemorragia Intracraniana (HIC) Grau IV.

A exclusão por instabilidade clínica e HIC grau IV ocorreu devido esses RN não poderem ser submetidos a avaliação neurocomportamental, já que o projeto principal ao qual este está vinculado avaliou o neurocomportamento desses RN.

\section{Resultados}

Dentre os 17 RNPT, 12 (70,6\%) são do sexo masculino e cinco $(29,4 \%)$ do sexo feminino. A IG dos RNPT variou com um mínimo de 25 semanas e máximo de 34 semanas (média = 32 semanas). O peso variou entre $640 \mathrm{~g}$ e $2.620 \mathrm{~g}$ com média de 1.636,4g (DP=499,5). A pontuação média para o SNAPPE II (Score for Neonatal Acute Physiology with Perinatal Extension version II) foi de 14,6 (DP=9,1), conforme está na tabela 1.

TABELA 1: Procedimentos realizados nos primeiros 14 dias de acordo com a idade gestacional. Rio de Janeiro, Brasil ( $n=17$ )

\begin{tabular}{cccc}
\hline $\begin{array}{c}\text { Idade Gestacional } \\
\text { (n) }\end{array}$ & $\begin{array}{c}\text { Procedimentos Dolorosos * } \\
\text { n (Média) }\end{array}$ & $\begin{array}{c}\text { Procedimentos Muito Dolorosos** } \\
\text { n (Média) }\end{array}$ & $\begin{array}{c}\text { Procedimentos Totais } \\
\text { n (Média) }\end{array}$ \\
\hline <32 semanas (3) & $204(68)$ & $72(24)$ & $276(92)$ \\
$>32$ semanas (14) & $227(16,2)$ & $226(16,1)$ & $453(32,3)$ \\
Total (17) & $431(25,3)$ & $298(17,5)$ & $729(42,9)$ \\
\hline
\end{tabular}

* Procedimentos Dolorosos (aspiração de vias aéreas superiores, re/inserção de pronga nasal, inserção de sonda gástrica e retirada de adesivos); **Procedimentos Muito Dolorosos (punção venosa, punção arterial, punção de calcâneo, aspiração de tubo orotraqueal, intubação orotraqueal e cateter central de inserção periférica).

O SNAPPE II avalia o paciente nas primeiras 12 horas de admissão na UTIN observando alterações fisiológicas múltiplas. A pontuação do SNAPPE II varia de zero a 162. Quanto maior a pontuação, maior a gravidade do $\mathrm{RN}^{10}$. 
Foi registrado um total de 729 procedimentos. Foram realizados 42,9 procedimentos por RNPT durante os primeiros 14 dias de nascido, totalizando aproximadamente três $(3,063)$ procedimentos realizados por RNPT no serviço diurno.

Identificou-se que quanto menor a IG dos RNPT maior a quantidade de procedimentos realizados nos primeiros 14 dias. Em RNPT com até 32 semanas de IG foram realizadas em média 92 ( $D P=1,4$ ) procedimentos dolorosos durante os primeiros 14 dias, já os RN com IG maior que 32 semanas a média foi de 32,3 procedimentos (DP=11,8) (Tabela 1).

Entretanto, para cada RNPT com IG menor que 32 semanas, foram realizados em média 16,7 manejos ao longo dos primeiros 14 dias, enquanto que para os RN com IG maior que 32 semanas foram realizados em média 5 manejos.

Entre os procedimentos, o mais frequente foi a punção de calcâneo (23,9\%), seguido da re/inserção de pronga $(20,2 \%)$, sondagem gástrica $(17,1 \%)$, aspiração de vias aéreas superiores $(12,8 \%)$, remoção de adesivo (8,6\%), aspiração de tubo orotraqueal $(5,2 \%)$, punção venosa $(5,1 \%)$, punção arterial $(4,5 \%)$ e passagem de cateter central de inserção periférica (2,1\%\%). O procedimento menos frequente foi a intubação orotraqueal $(0,5 \%)$.

Dos 729 procedimentos realizados, apenas 124 receberam algum tipo manejo (um ou mais), os demais 605 procedimentos não receberam nenhum manejo. O manejo predominante foi a contenção facilitada, com um total de 53 (32,7\%) (Tabela 2). Dos 37 procedimentos de punção venosa, em 16 (43,2\%) houve mais de um manejo realizado, desses 16 casos, 12 (75\%) tiveram a associação de sucção não nutritiva com solução adocicada.

TABELA 2: Manejo da Dor para Cada Tipo de Procedimento Doloroso e Total de Procedimentos que não Receberam Nenhum Manejo. Rio de Janeiro, Brasil $(n=17)$

\begin{tabular}{|c|c|c|c|c|c|c|}
\hline Procedimento & $\begin{array}{c}\text { Contenção } \\
\text { n (\%) }\end{array}$ & $\begin{array}{l}\text { SNN* } \\
\text { n (\%) }\end{array}$ & $\begin{array}{c}\text { Solução } \\
\text { Adocicada } \\
\text { n (\%) }\end{array}$ & $\begin{array}{l}\text { Fentanil } \\
\text { n (\%) }\end{array}$ & $\begin{array}{c}\text { Total de } \\
\text { Manejos } \\
\mathbf{n}\end{array}$ & $\begin{array}{c}\text { Procedimento } \\
\text { Sem Manejo } \\
n\end{array}$ \\
\hline Punção Venosa & $6(13,6)$ & $19(43,2)$ & $19(43,2)$ & - & 44 & 14 \\
\hline Punção de Calcâneo & $8(26,7)$ & $7(23,3)$ & $15(50)$ & - & 30 & 156 \\
\hline Punção Arterial & $9(39,1)$ & $7(30,4)$ & $9(39,1)$ & - & 25 & 17 \\
\hline Aspiração de VAS* & $10(62,5)$ & $5(31,25)$ & $1(6,25)$ & - & 16 & 77 \\
\hline PICC* & - & - & - & $15(100)$ & 15 & - \\
\hline SNG/SOG* & $3(25)$ & $4(33,3)$ & $5(41,7)$ & - & 12 & 115 \\
\hline Re/Inserção de Pronga & $10(100)$ & - & - & - & 10 & 135 \\
\hline Retirada de Adesivo & $3(60)$ & $1(20)$ & $1(20)$ & - & 5 & 59 \\
\hline Aspiração de TOT* & $4(80)$ & $1(20)$ & - & - & 5 & 29 \\
\hline $\mathrm{IOT}^{*}$ & - & - & - & $1(100)$ & 1 & 3 \\
\hline Total & $53(32,5)$ & $44(27)$ & $50(30,7)$ & $16(9,8)$ & 163 & 605 \\
\hline
\end{tabular}

*SNN (Sucção Não Nutritiva); SOG (Sonda Orográstica); SNG (Sonda Nasogástrica); VAS (Vias Aéreas Superiores); TOT (Tubo Orotraqueal); PICC (Cateter Central de Inserção Periférica); IOT (Intubação Orotraqueal).

\section{DISCUSSÃo}

O presente estudo registrou um total de 729 procedimentos dolorosos, com uma média de aproximadamente três procedimentos realizados por RN no serviço diurno.

Estudo realizado com 89 prematuros em um hospital escola do interior paulista identificou que cada RN foi exposto à média de 75,1 procedimentos dolorosos durante os primeiros 14 dias, resultando em média diária de 5,4 procedimentos por prematuro ${ }^{12}$. Este estudo utilizou uma tabela com 13 procedimentos a mais que o utilizado nesta pesquisa. Além disso, foram quantificados os procedimentos também realizados no serviço noturno e maior número de participantes. Logo, a quantidade reduzida de procedimentos pré-determinados para a quantificação, a limitação da coleta de dados que ocorreu apenas no serviço diurno e a amostra ter sido pequena pode justificar a quantidade diária de procedimentos realizados por RN apresentar-se inferior nesta pesquisa.

A coleta dos procedimentos dolorosos apresentou limites, por ser recordatória. A ficha com a quantidade de procedimentos realizados durante o plantão era preenchida no final do dia, sendo necessário que o profissional recordasse dos procedimentos que o mesmo realizou no RNPT incluso na pesquisa. Este também pode ter sido um fator contribuinte para um valor inferior em relação à quantidade dos procedimentos dolorosos. 
A intubação orotraqueal foi o procedimento com menor frequência. Contudo, a contagem dos procedimentos dolorosos começava a partir da internação do RNPT nas unidades neonatais, o que excluía as intubações realizadas ainda no Centro Obstétrico.

A punção de calcâneo ainda constitui o método de escolha para coleta de amostra de sangue na maioria das unidades neonatais e representa o evento doloroso mais comum entre todos os procedimentos realizados em recém-nascidos ${ }^{13}$.

O uso generalizado da punção de calcâneo nos recém-nascidos está associado principalmente à sua estrutura arteriovenosa limitada, restringindo assim o uso da punção venosa; realizar uma punção no calcâneo é um procedimento fácil que pode ser conduzido por uma enfermeira sozinha, o que representa uma situação comum em muitas enfermarias neonatais ${ }^{14}$.

Além disso, a frequência da punção de calcâneo justifica-se por ser um procedimento realizado para identificação da dosagem glicêmica do RN, e este, ser um dos exames frequentemente realizado nas unidades neonatais. Um recurso para diminuir a quantidade excessiva da punção de calcâneo é a comunicação efetiva por toda equipe multidisciplinar para quando for necessária a coleta venosa ou arterial para outros exames utilizar a mesma amostra de sangue para realizar também o teste glicêmico.

Com o intuito de diminuir a frequência desse procedimento nas unidades neonatais, é proposto o uso de dispositivos que possam realizar várias análises $(\mathrm{pH}, \mathrm{PaO} 2, \mathrm{PaCO} 2$, eletrólitos, cálcio, bilirrubina, lactato) a partir de uma única amostra pequena de sangue, reduzindo assim o número de punções de calcâneo necessários para testes de laboratório. Além disso, também se indica que sejam colocados cateteres venosos arteriais ou centrais periféricos em pacientes que precisam de mais de 3-4 punções por dia. Esses procedimentos devem ser realizados com analgesia adequada ${ }^{15}$.

Apesar da punção de calcâneo ter sido o procedimento mais frequente, apenas em 10,3\% foi realizado algum tipo de manejo. Esse dado pode nos apontar que o manejo da dor referente a procedimentos corriqueiros na unidade neonatal ainda é desvalorizado.

Uma metanálise recente revelou que a glicose é uma alternativa aceitável à sacarose, diminuindo os escores de PIPP e os tempos de choro associados à punção venosa e à punção do calcâneo ${ }^{16}$.

Outra maneira eficaz é a associação de métodos que visem o alívio da dor durante procedimentos dolorosos agudo. Um estudo prospectivo, randomizado, realizado com 102 recém-nascidos identificou que para os bebês que receberam sucção + leite materno + contenção e sucção + leite materno durante os procedimentos de punção de calcâneo, as chances de dor moderada a intensa foram $87,4 \%$ e $95,7 \%$ inferiores às dos bebês que receberam cuidados de rotina, respectivamente ${ }^{17}$.

A (re)inserção de pronga chama atenção por ter sido o segundo procedimento mais realizado. Em contrapartida, em apenas 10 dos 147 procedimentos houve algum manejo, e o único realizado para este procedimento foi a contenção facilitada.

É importante haver a compreensão da maneira adequada de realizar a contenção facilitada, de modo a oferecer aconchego durante o procedimento doloroso, e não apenas uma contenção restritiva de movimentos do RN para facilitar a realização do procedimento.

Mudar o RN de posição, aninhar, enrolar no cueiro, manter posição flexionada e suporte postural com contenção manual tem sido recomendado para facilitar a organização e autorregulação dos neonatos, durante a dor aguda ${ }^{18}$. Devese fazer o enrolamento antes do procedimento doloroso, utilizando uma coberta ou cueiro, envolvendo o neonato com a flexão das extremidades inferiores, e alinhando na linha mediana dos membros superiores flexionados, posicionando a mão perto da boca ${ }^{19}$.

Além da contenção facilitada, existem várias técnicas não farmacológicas que podem ser utilizadas para aliviar a dor dos recém-nascidos, como a sucção não nutritiva, posição canguru, colo materno, a presença do ninho, amamentação, musicoterapia e estimulação olfativa. A integração dessas intervenções na rotina clínica aliviará o sofrimento neonatal e proporcionará maior satisfação dos pais e da equipe clínica ${ }^{20}$.

A sucção não nutritiva, sacarose oral e contenção facilitada parecem ser as intervenções não farmacológica mais promissoras para alívio da dor, durante a utilização do CPAP nasal. Um estudo randomizado e controlado, envolvendo 30 RNPT indicou que ao instalar o CPAP nasal 100\% dos RNPTs sentem dor, no entanto quando oferece a suç̧ão não nutritiva, os RNPTs reagem ao estímulo sem atingir uma pontuação indicativa de dor, de acordo com a NIPS ${ }^{21}$.

Uma meta-análise de 57 estudos, incluindo mais de 4730 lactentes com idade gestacional variando de 25 a 44 semanas, concluiu que a sacarose é segura e eficaz para reduzir a dor processual de um único evento. Foram observadas reduções máximas nos indicadores de dor fisiológica e comportamental quando a sacarose foi administrada 2 minutos antes de um estímulo doloroso e os efeitos duraram 4 minutos ${ }^{22}$. 
A combinação de sucção não nutritiva e glicose tem se mostrado eficaz na redução da proporção de tempo de choro após procedimentos dolorosos simples e, em alguns casos, reduz os indicadores comportamentais e fisiológicos da dor ${ }^{23}$.

O adesivo é um dispositivo frequentemente utilizado nas unidades neonatais, porém sua retirada além de ser potencialmente dolorosa pode desencadear lesões de pele no RNPT. A pele do RN apresenta algumas peculiaridades em relação à do adulto, como: é mais fina (40 a 60\%), menos pilosa, com menor coesão entre a epiderme e a derme, e a proporção entre a área de superfície corpórea e o peso é de cinco vezes a do adulto ${ }^{24}$.

A lesão da pele é comum nos RNPT que se encontram hospitalizados e logo no primeiro mês de vida cerca de $80 \%$ dessas crianças já sofreram alguma lesão na pele. A utilização de adesivos utilizados em UTIN para fixar materiais como cateter venoso, tubo endotraqueal e outros que podem aderir-se fortemente à pele, chegando a arrancar as camadas superficiais ou mesmo toda a epiderme ao serem removidos. Deve-se usar uma quantidade mínima de esparadrapo ou adesivo, colocando protetores à base de material suave entre estes fixadores e a pele $e^{25}$.

Mesmo se tratando de uma população mais suscetíveis à lesão de pele, e consequentemente com mais exposição à dor, a remoção de adesivos contou com apenas 5 manejos para um total de 63 procedimentos realizados nesse período de coleta de dados.

A fim de evitar lesões de pele em RN, o uso de adesivos deve ser limitado e discriminado. Sua retirada deve ser feita de maneira cuidadosa, utilizando-se gaze umedecida em solução salina, possibilitando remoção atraumática ${ }^{26}$.

É de suma importância que haja o manejo adequado da dor para retirada de adesivos, entendendo que é um procedimento usualmente realizado nas unidades neonatais e que submete o recém-nascido a um processo doloroso.

É importante destacar que a inclusão do procedimento de remoção de adesivo no instrumento do estudo ocorreu devido à sugestão da própria equipe por considerar este um procedimento doloroso. Entretanto, em apenas $6,3 \%$ houve algum manejo, indicando distanciamento entre o conhecimento do processo doloroso causado ao RN pelo procedimento e a iniciativa em realizar o manejo da dor.

Os profissionais que realizam procedimentos invasivos de modo rotineiro podem ter sua sensibilidade diminuída por meio de uma reestruturação cognitiva, tornando-se mais céticos em relação às respostas subjetivas de dor exibidas pelas crianças ${ }^{27,28}$.

É necessário entender o quão o ambiente pode influenciar na prática assistencial dos profissionais, encorajandoos ou não na aplicação do conhecimento no cenário clínico e na busca pela assistência de qualidade.

Nesse ponto, a cultura organizacional será uma variável importante a ser considerada na análise do engajamento desses profissionais. Entende-se por cultura organizacional, os valores, crenças, costumes e normas compartilhadas pelos membros de uma organização e pode ser classificada em vários tipos, como cultura orientada a hierarquia, inovação, tarefa, entre outros ${ }^{29}$.

$\mathrm{Na}$ instituição em que foi realizado o estudo já existe um protocolo clínico de dor implementado e houve capacitação de toda equipe multiprofissional da unidade, sobre avaliação, utilização de escala de dor, sendo instituída a NIPS (Neonatal Infant Pain Scale) no setor como avaliação diária de todos RN internados, e condutas adequadas de manejo da dor. Todavia, a capacitação ocorreu no ano de 2015, e ao longo desse período a equipe foi renovada com mudanças de profissionais que não receberam treinamento completo, o que indica a necessidade de capacitação constante de toda equipe para que o conhecimento e sua aplicação na prática sejam contínuos.

\section{CONCLUSÃO}

O presente estudo permitiu apreender que a despeito da quantidade de procedimentos dolorosos agudos realizados no RNPT durante seus primeiros 14 dias de internação em uma unidade neonatal, o manejo da dor ainda é um desafio.

Espera-se que o estudo incite não apenas à reflexão da atuação do profissional frente aos procedimentos dolorosos, mas também leve à mudança de posicionamento quanto à realização de manejos que visam a prevenção e alívio da dor desses bebês já fragilizados pela patologia de base e pelo ambiente estressor da UTI.

Um caminho para tal mudança pode também decorrer de implementações de protocolos de avaliação e tratamento da dor em unidades neonatais, além de formulações de políticas públicas em saúde que visem à capacitação, aprimoramento e incentivo de instituições e profissionais a adotarem no cenário clínico ações que minimizem o impacto da dor nos neonatos internados.

Cabe ressaltar ainda a necessidade de explorar cada vez mais esse tema através de produções científicas para contribuir com melhoria na atuação prática nas unidades neonatais. 


\section{REFERÊNCIAS}

1. Kassab M, Alhassan AA, Alzoubi KH, Khader YS. Number and frequency of routinely applied painful procedures in university neonatal intensive care unit. Clinical Nursing Research. 2017 [cited 2019 Aug 15]; 1:1-14. DOI: https://doi.org/10.1177/1054773817744324

2. Roofthooft DWE, Simons SHP, Anand KJS, Tibboel D, van Dijk M. Eight Years Later, Are We Still Hurting Newborn Infants? Neonatology. 2014 [cited 2019 Aug 15]; 105:218-226. DOI: https://doi.org/10.1159/000357207

3. Bhalla T, Shepherd E, Tobias JD. Neonatal pain management. Saudi J Anaesth. 2014; 8:89-97. Available from: https://www.ncbi.nlm.nih.gov/pmc/articles/PMC4268538/

4. Moultrie F, Slater R, Hartley C. Improving the treatment of infant pain. Curr Opin Support palliat Care (Oxford). 2017 [cited 2019 Aug 15]; 11:112-17. Available from: https://www.ncbi.nlm.nih.gov/pmc/articles/PMC5419813/pdf/cospa-11-112.pdf

5. Prevention and management of procedural pain in the neonate: An update, American Academy of Pediatrics. COMMITTEE ON FETUS AND NEWBORN and SECTION ON NESTHESIOLOGY AND PAIN MEDICINE. Pediatrics. 2016;137. [cited 2019 Aug 15]. DOI: https://doi.org/10.1542/peds.2015-4271

6. Badr LK, Abdallah B, Kahale L. A meta-analysis of preterm infant massage: An ancient practice with contemporary applications. MCN American Journal of Maternal Child Nursing. 2015 [cited 2019 Aug 15]; 40:344-358. Available from: https://www.ncbi.nlm.nih.gov/pubmed/26302088

7. Foster JP, Psaila K, Patterson T. Non-nutritive sucking for increasing physiologic stability and nutrition in preterm infants. Cochrane Database Systematic Reviews. 2016 [cited 2019 Aug 15]; 10. Available from: https://www.ncbi.nlm.nih.gov/pmc/articles/PMC6458048/pdf/CD001071.pdf

8. Johnston C, Campbell-Yeo M, Disher T, Benoit B, Fernandes A, Streiner D, et. al. Skin-to-skin care for procedural pain in neonates. Cochrane Database of Systematic Reviews. 2017 [cited 2019 Aug 15]; 2. DOI: https://doi.org/10.1002/14651858.CD008435.pub2

9. Norina Witt, Seth Coynor, Christopher Edwards, and Hans Bradshaw. A Guide to Pain Assessment and Management in the Neonate. Curr Emerg Hosp Med Rep. 2016 [cited 2019 Aug 15]; 4:1-10. Available from: https://www.ncbi.nlm.nih.gov/pmc/articles/PMC4819510/pdf/40138_2016_Article_89.pdf

10. Cignacco E, Hamers J, Lingen RA, Stoffel L, Büchi S, Müller R et al. Neonatal procedural pain exposure and pain management in ventilated preterm infants during the first 14 days of life. Swiss Med Wkly. 2009 [cited 2019 Aug 15]; 139:226-32. Available from: http://portaldeboaspraticas.iff.fiocruz.br/wp-content/uploads/2017/09/CIGNACCO-Eva-HAMERSB-Jan-LINGENC-RichardA.-van-et-al..pdf

11. Harsha SS, Archana BR. SNAPPE-II (Score for Neonatal Acute Physiology with Perinatal Extension-II) in Predicting Mortality and Morbidity in NICU. J Clin Diagn Res. 2015 [cited 2019 Aug 15]; 9:SC10-SC12. DOI: https://doi.org/10.1067/mpd.2001.109608

12. Bonutti DP, Daré MF, Castral TC, Leite AM, Vici-Maia JÁ, Scochi CGS. Dimensionamento dos procedimentos dolorosos e intervenções para alívio da dor aguda em prematuros. Rev Latino-Am Enferm. 2017 [cited 2019 Aug 15]; 25:1-9. DOI: http://dx.doi.org/10.1590/1518-8345.1387.2917

13. Carbajal R, Rousset A, Danan C, Couquery S, Nolent $P$, Ducrocq $S$ et al. Epidemiology and treatment of painful procedures in neonates in intensive care units. JAMA. 2008 [cited 2019 Aug 15]; 300:60-70.

14. Sorrentinoa G, Fumagallia M, Milanib I, Cortinovisb A, Zorza G, Cavallaroa F et al. The impact of automatic devices for capillary blood collection on efficiency and pain response in newborns: A randomized controlled trial. Int J Nurs Stud. 2017 [cited 2019 Aug 15]; 72:24-9. DOI: https://doi.org/10.1016/j.ijnurstu.2017.04.001

15. Hall RW, Anand KJS. Pain Management in Newborns. Clin Perinatol. 2014 [cited 2019 Aug 15]; 41: 895-924. Available from: https://www.ncbi.nlm.nih.gov/pmc/articles/PMC4254489/pdf/nihms626365.pdf

16. Bueno M, Yamada J, Harrison D, Khan S, Ohlsson A, Adams-Webber T et. al. A systematic review and meta-analyses of nonsucrose sweet solutions for pain relief in neonates. Pain Research and Management. 2013 [cited 2019 Aug 15]; 18(3):15361. DOI: http://dx.doi.org/10.1155/2013/956549

17. Peng H-F, Yin T, Yang L, Wang C, Chang Y-C, Jeng M-J et. al. Non-nutritive sucking, oral breast milk, and facilitated tucking relieve preterm infant pain during heel-stick procedures: A prospective, randomized controlled trial. International Journal of Nursing Studies. 2018 [cited 2019 Aug 15]; 77:162-170. DOI: https://doi.org/10.1016/j.ijnurstu.2017.10.001

18. Gaspardo CM, Linhares MBM, Martinez FE. The efficacy of sucrose for the relief of pain in neonates: a systematic review of the literature. J Pediatr. 2005 [cited 2019 Aug 15]; 81:435-42. DOI: http://dx.doi.org/10.2223/JPED.1417

19. Tamez RN. Controle da dor e sedação no neonato. Enfermagem na UTI Neonatal: assistência ao recém-nascido de alto risco. 6th ed. Rio de Janeiro: Guanabara Koogan; 2016.

20. Hasnaa S, Asharaf E, Hesham A. Does Topical Lidocaine Reduce the Pain Associated with the Insertion of Nasal Continuous Positive Airway Pressure Prongs in Preterm Infants? The Clinical Journal of Pain. 2016 [cited 2019 Aug 15]; 32 (11): $948-957$. Available from: https://insights.ovid.com/crossref?an=00002508-201611000-00005

21. Antunes JCP, Nascimento MPA. The non-nutritive sucking of premature newborn as a nursing technology. Rev bras enferm. 2013 [cited 2019 Aug 15]; 66:663-7. Available from: http://www.scielo.br/pdf/reben/v66n5/04.pdf

22. Stevens B, Yamada J, Ohlsson A, Haliburton S, Shorkey A. Sucrose for analgesia in newborn infants undergoing painful procedures. Cochrane Database of Systematic Reviews. 2016; [cited 2019 Aug 15]. 7. Available from: https://www.ncbi.nlm.nih.gov/pmc/articles/PMC6457867/pdf/CD001069.pdf 
23. Witt N, Coynor S, Edwards C, Bradshaw H. A Guide to Pain Assessment and Management in the Neonate. Curr Emerg Hosp Med Rep. 2016 [cited 2019 Aug 15]; 4:1-10. Available from: https://www.ncbi.nlm.nih.gov/pmc/articles/PMC4819510/pdf/40138_2016_Article_89.pdf

24. Meszes A, Tálosi G, Máder K, Orvos H, Kemény L, Csoma ZR. Lesions requiring wound management in a central tertiary neonatal intensive care unit. World J Pediatr. 2017 [cited 2019 Aug 15]; 13(2):165-172. Available from: https://link.springer.com/article/10.1007\%2Fs12519-016-0070-6

25. Adriano LSM, Freire ILS, Pinto JTJM. Intensive care with the skin of newborn pre-term. Rev Eletr Enf. 2009 [cited 2019 Aug 15]; 11:173- 80. Available from: http://www.fen.ufg.br/revista/v11/n1/v11n1a22.htm.

26. Meszes A, Tálosi G, Máder K, Orvos H, Kemény L, Csoma ZR. Lesions requiring wound management in a central tertiary neonatal intensive care unit. World J Pediatr. 2017 [cited 2019 Aug 15]; 13(2):165-172. Available from: https://link.springer.com/article/10.1007\%2Fs12519-016-0070-6

27. Maia ACA, Coutinho SB. Factors that influence the practice of healthcare professionals regarding pain management in newborn infants. Rev Paul de Pediatr. 2011 [cited 2019 Aug 15]; 29:270-76. Available from: http://www.scielo.br/pdf/rpp/v29n2/a20v29n2.pdf

28. Borghesan NBAlves et al. Peripherally inserted central catheter: practics of nursing team in the neonatal intensive care. Rev enferm UERJ. [Internet] 2017; 25:e28143. [cited Oct 5 2019]. DOI: https://doi.org/10.12957/reuerj.2017.28143.

29. An Y, Kang J. Relationship between Organizational Culture and Workplace Bullyingamong Korean Nurses. Asian Nursing Research. 2016 [cited 2019 Aug 15]; 10: 234-239. DOI: http://dx.doi.org/10.1016/j.anr.2016.06.004 